The Institutional Repository IRUA is a subset of the Academic bibliography. As of 1991 the UA library produces an academic bibliography for the University of Antwerp (UA). IRUA contains scientific publications of UA researchers. If allowed the fulltext of the publications are made available.

This paper was published in 'Optical materials express' and is made available as an electronic reprint with the permission of OSA. The paper can be found at the following URL on the OSAwebsite: http://dx.doi.org/doi:10.1364/OME.1.000551.

Systematic or multiple reproduction or distribution to multiple locations via electronic or other means is prohibited and is subject to penalties under law. 


\title{
Rapid, broadband two-photon-excited fluorescence spectroscopy and its application to red-emitting secondary reference compounds
}

\author{
Nikolay S. Makarov, ${ }^{1}$ Jochen Campo,${ }^{1,2}$ Joel M. Hales, ${ }^{1}$ and Joseph W. Perry ${ }^{1, *}$ \\ ${ }^{I}$ School of Chemistry and Biochemistry, Georgia Institute of Technology, 901 Atlantic Drive, Atlanta, GA 30332, USA \\ ${ }^{2}$ Department of Physics, University of Antwerp (campus Drie Eiken), Universiteitsplein 1, B-2610 Antwerpen, \\ Belgium \\ *joe.perry@gatech.edu
}

\begin{abstract}
We present a methodology for rapidly acquiring broadband twophoton absorption (2PA) spectra by means of a compact two-channel femtosecond fluorescence excitation apparatus. This technique is insensitive to differences in excitation and collection geometries as well as detection efficiencies between the sample and reference, in addition to variations in average power, pulse duration and spatial beam profile, as it utilizes sequential measurement of the sample and reference in each of the two cell positions. Our approach eliminates the need to determine the fluorescence quantum yields of the sample and reference, as it allows measurement of emission from samples at a common specified wavelength. These attributes allow for acquisition of 2PA spectra with an estimated accuracy of $\pm 15 \%$ (limited almost exclusively by the uncertainty in the 2PA cross section for the reference standards) over an excitation range of 550-1600 nanometers with a typical time per spectrum of $\sim 30-60$ minutes. We have applied this technique to determine the $2 \mathrm{PA}$ spectra of six commercially available organic dyes over a wide range of excitation wavelengths $(670-1600 \mathrm{~nm})$, which can be used as secondary reference standards emitting in the red and near-infrared spectral region $(600-1000 \mathrm{~nm})$. We have also characterized some of these compounds using the femtosecond-pulsed Z-scan method and found very good agreement with the fluorescence-based measurements.
\end{abstract}

(C)2011 Optical Society of America

OCIS codes: (190.0190) Nonlinear optics; (300.6410) Spectroscopy, multiphoton; (190.4710)

Optical nonlinearities in organic materials.

\section{References and links}

1. D. A. Parthenopoulos and P. M. Rentzepis, "Three-dimensional optical storage memory," Science 245(4920), 843-845 (1989).

2. N. Makarov, A. Rebane, M. Drobizhev, H. Wolleb, and H. Spahni, "Optimizing two-photon absorption for volumetric optical data storage," J. Opt. Soc. Am. B 24(8), 1874-1885 (2007) (and references therein).

3. J. H. Strickler and W. W. Webb, "3-D optical data storage by two-photon excitation," Adv. Mater. (Deerfield Beach Fla.) 5(6), 479-481 (1993).

4. R. R. Birge, B. Parsons, Q. W. Song, and J. R. Tallent, "Protein-based three-dimensional memories and associative processors," in Molecular Electronics, M.A. Ratner and J. Jortner, eds. (Blackwell Science, 1997).

5. S. Kawata and Y. Kawata, "Three-dimensional optical data storage using photochromic materials," Chem. Rev. 100(5), 1777-1788 (2000).

6. J. D. Bhawalkar, N. D. Kumar, C. F. Zhao, and P. N. Prasad, “Two-photon photodynamic therapy,” J. Clin. Laser Med. Surg. 15(5), 201-204 (1997).

7. A. Karotki, M. Kruk, M. Drobizhev, A. Rebane, E. Nickel, and C. W. Spangler, "Efficient singlet oxygen generation upon two-photon excitation of new porphyrin with enhanced nonlinear absorption," IEEE J. Sel. Top. Quantum Electron. 7(6), 971-975 (2001).

8. H. A. Collins, M. Khurana, E. H. Moriyama, A. Mariampillai, E. Dahlstedt, M. Balaz, M. K. Kuimova, M. Drobizhev, V. X. D. Yang, D. Phillips, A. Rebane, B. C. Wilson, and H. L. Anderson, "Blood-vessel closure using photosensitizers engineered for two-photon excitation," Nat. Photonics 2(7), 420-424 (2008).

9. J. R. Starkey, A. K. Rebane, M. A. Drobizhev, F. Meng, A. Gong, A. Elliott, K. McInnerney, and C. W. Spangler, "New two-photon activated photodynamic therapy sensitizers induce xenograft tumor regressions after near-IR laser treatment through the body of the host mouse," Clin. Cancer Res. 14(20), 6564-6573 (2008). 
10. T. Karu and V. Letokhov, "Possible benefits of two-quantum excitation in ALA-PDT?" J. Photochem. Photobiol. B 23(2-3), 261-262 (1994).

11. D. Leupold and I. E. Kochevar, "Multiphoton photochemistry in biological systems. Introduction," Photochem. Photobiol. 66(5), 562-565 (1997).

12. D. Gao, R. R. Agayan, H. Xu, M. A. Philbert, and R. Kopelman, "Nanoparticles for two-photon photodynamic therapy in living cells," Nano Lett. 6(11), 2383-2386 (2006)

13. C. W. Spangler, "Recent development in the design of organic materials for optical power limiting," J. Mater. Chem. 9(9), 2013-2020 (1999).

14. J. M. Hales, M. Cozzuol, T. E. O. Screen, H. L. Anderson, and J. W. Perry, "Metalloporphyrin polymer with temporally agile, broadband nonlinear absorption for optical limiting in the near infrared," Opt. Express 17(21), 18478-18488 (2009).

15. G. S. He, J. D. Bhawalkar, C. F. Zhao, and P. N. Prasad, "Optical limiting effect in a two-photon absorption dye doped solid matrix," Appl. Phys. Lett. 67(17), 2433-2435 (1995).

16. D. I. Kovsh, S. Yang, D. J. Hagan, and E. W. Van Stryland, "Nonlinear optical beam propagation for optical limiting," Appl. Opt. 38(24), 5168-5180 (1999).

17. J. Oberlé, L. Bramerie, G. Jonusauskas, and C. Rullière, "Optical-limiting properties of a push-pull diphenylbutadiene," Opt. Commun. 169(1-6), 325-332 (1999).

18. Y. Morel, A. Ibanez, C. Nguefack, C. Andraud, A. Collet, J.-F. Nicoud, and P. L. Baldeck, "Nonlinear absorption spectra of transparent organic crystals for optical limiting applications at visible wavelengths," Synth. Met. 115(1-3), 265-268 (2000).

19. J. E. Ehrlich, X. L. Wu, I. Y. S. Lee, Z. Y. Hu, H. Röckel, S. R. Marder, and J. W. Perry, "Two-photon absorption and broadband optical limiting with bis-donor stilbenes," Opt. Lett. 22(24), 1843-1845 (1997).

20. W. R. Zipfel, R. M. Williams, and W. W. Webb, "Nonlinear magic: multiphoton microscopy in the biosciences," Nat. Biotechnol. 21(11), 1369-1377 (2003).

21. M. Drobizhev, S. Tillo, N. S. Makarov, T. E. Hughes, and A. Rebane, "Absolute two-photon absorption spectra and two-photon brightness of orange and red fluorescent proteins," J. Phys. Chem. B 113(4), 855-859 (2009).

22. B. R. Masters, P. T. C. So, and E. Gratton, "Multiphoton excitation fluorescence microscopy and spectroscopy of in vivo human skin," Biophys. J. 72(6), 2405-2412 (1997).

23. R. Gauderon and C. J. R. Sheppard, "Effect of a finite-size pinhole on noise performance in single-, two-, and three-photon confocal fluorescence microscopy," Appl. Opt. 38(16), 3562-3565 (1999).

24. G. H. Patterson and D. W. Piston, "Photobleaching in two-photon excitation microscopy," Biophys. J. 78(4), 2159-2162 (2000).

25. Y. Imanishi, K. H. Lodowski, and Y. Koutalos, "Two-photon microscopy: shedding light on the chemistry of vision," Biochemistry 46(34), 9674-9684 (2007).

26. J. W. Perry, B. H. Cumpston, S. P. Ananthavel, S. Barlow, D. L. Dyer, J. E. Ehrlich, L. L. Erskine, A. A. Heikal, S. M. Kuebler, I.-Y. S. Lee, D. McCord-Maughon, J. Qin, H. Röckel, M. Rumi, X.-L. Wu, and S. R. Marder, "Two-photon polymerization initiators for three-dimensional optical data storage and microfabrication," Nature 398(6722), 51-54 (1999)

27. S. Maruo and S. Kawata, "Two-photon-absorbed near-infrared photopolymerization for three-dimensional microfabrication,” J. Microelectromech. Syst. 7(4), 411-415 (1998).

28. K. D. Belfield, K. J. Schafer, Y. Liu, J. Liu, X. Ren, and E. W. V. Stryland, "Multiphoton-absorbing organic materials for microfabrication, emerging optical applications and non-destructive three-dimensional imaging," J. Phys. Org. Chem. 13(12), 837-849 (2000).

29. H.-B. Sun, T. Kawakami, Y. Xu, J.-Y. Ye, S. Matuso, H. Misawa, M. Miwa, and R. Kaneko, "Real threedimensional microstructures fabricated by photopolymerization of resins through two-photon absorption," Opt. Lett. 25(15), 1110-1112 (2000).

30. M. Miwa, S. Juodkazis, T. Kawakami, S. Matsuo, and H. Misawa, "Femtosecond two-photon stereolithography,” Appl. Phys., A Mater. Sci. Process. 73(5), 561-566 (2001).

31. I. Wang, M. Bouriau, P. L. Baldeck, C. Martineau, and C. Andraud, "Three-dimensional microfabrication by two-photon-initiated polymerization with a low-cost microlaser," Opt. Lett. 27(15), 1348-1350 (2002).

32. W. Haske, V. W. Chen, J. M. Hales, W. Dong, S. Barlow, S. R. Marder, and J. W. Perry, " $65 \mathrm{~nm}$ feature sizes using visible wavelength 3-D multiphoton lithography," Opt. Express 15(6), 3426-3436 (2007).

33. N. S. Makarov, M. Drobizhev, and A. Rebane, "Two-photon absorption standards in the 550-1600 nm excitation wavelength range," Opt. Express 16(6), 4029-4047 (2008)

34. C. Xu and W. W. Webb, "Measurement of two-photon excitation cross sections of molecular fluorophores with data from 690 to 1050 nm," J. Opt. Soc. Am. B 13(3), 481-491 (1996).

35. M. A. Albota, C. Xu, and W. W. Webb, "Two-photon fluorescence excitation cross sections of biomolecular probes from 690 to $960 \mathrm{~nm}$," Appl. Opt. 37(31), 7352-7356 (1998).

36. D. Beljonne, W. Wenseleers, E. Zojer, Z. G. Shuai, H. Vogel, S. J. K. Pond, J. W. Perry, S. R. Marder, and J. L. Bredas, "Role of dimensionality on the two-photon absorption response of conjugated molecules: the case of octupolar compounds," Adv. Funct. Mater. 12(9), 631-641 (2002).

37. M. Albota, D. Beljonne, J. L. Brédas, J. E. Ehrlich, J. Y. Fu, A. A. Heikal, S. E. Hess, T. Kogej, M. D. Levin, S. R. Marder, D. McCord-Maughon, J. W. Perry, H. Röckel, M. Rumi, G. Subramaniam, W. W. Webb, X. L. Wu, and C. Xu, "Design of organic molecules with large two-photon absorption cross sections," Science 281(5383), 1653-1656 (1998).

38. M. Rumi, J. E. Ehrlich, A. A. Heikal, J. W. Perry, S. Barlow, Z. Y. Hu, D. McCord-Maughon, T. C. Parker, H. Rockel, S. Thayumanavan, S. R. Marder, D. Beljonne, and J. L. Bredas, "Structure-property relationships for 
two-photon absorbing chromophores: Bis-donor diphenylpolyene and bis(styryl)benzene derivatives," J. Am. Chem. Soc. 122(39), 9500-9510 (2000).

39. M. Rumi, S. J. K. Pond, T. Meyer-Friedrichsen, Q. Zhang, M. Bishop, Y. Zhang, S. Barlow, S. R. Marder, and J. W. Perry, "Tetrastyrylarene derivatives: comparison of one- and two-photon spectroscopic properties with distyrylarene analogues," J. Phys. Chem. C 112(21), 8061-8071 (2008).

40. M. Rumi and J. W. Perry, "Two-photon absorption: an overview of measurements and principles," Adv. Opt. Photon. 2(4), 451-518 (2010).

41. J. M. Hales, J. Matichak, S. Barlow, S. Ohira, K. Yesudas, J.-L. Brédas, J. W. Perry, and S. R. Marder, "Design of polymethine dyes with large third-order optical nonlinearities and loss figures of merit," Science 327(5972), 1485-1488 (2010).

42. H.-C. Lin, H. Kim, S. Barlow, J. M. Hales, J. W. Perry, and S. R. Marder, "Synthesis and linear and nonlinear optical properties of metal-terminated bis(dioxaborine) polymethines," Chem. Commun. (Camb.) 47(2), 782-784 (2010).

43. M. Drobizhev, S. Tillo, N. S. Makarov, T. E. Hughes, and A. Rebane, "Color hues in red fluorescent proteins are due to internal quadratic Stark effect," J. Phys. Chem. B 113(39), 12860-12864 (2009).

44. K. D. Belfield, S. Yao, and M. V. Bondar, "Organic multiphoton absorbing materials and devices," in Introduction to Organic Electronic and Optoelectronic Materials and Devices, S.-S. Sun and L.R. Dalton, eds. (CRC Press, 2008), pp. 573-606.

45. M. Rumi, J. E. Ehrlich, A. A. Heikal, J. W. Perry, S. Barlow, Z. Hu, D. McCord-Maughon, T. C. Parker, H. Röckel, S. Thayumanavan, S. R. Marder, D. Beljonne, and J.-L. Brédas, "Structure-property relationships for two-photon absorbing chromophores: bis-donor diphenylpolyene and bis(styryl)benzene derivatives," J. Am. Chem. Soc. 122(39), 9500-9510 (2000).

46. S. J. K. Pond, M. Rumi, M. D. Levin, T. C. Parker, D. Beljonne, M. W. Day, J.-L. Brédas, S. R. Marder, and J. W. Perry, "One- and two-photon spectroscopy of donor-acceptor-donor di(styryl)benzene derivatives: Effect of cyano substitution and distortion from planarity," J. Phys. Chem. A 106(47), 11470-11480 (2002).

47. S.-J. Chung, S. Zheng, T. Odani, L. Beverina, J. Fu, L. A. Padilha, A. Biesso, J. M. Hales, X. Zhan, K. Schmidt, A. Ye, E. Zojer, S. Barlow, D. J. Hagan, E. W. Van Stryland, Y. Yi, Z. Shuai, G. A. Pagani, J.-L. Brédas, J. W. Perry, and S. R. Marder, "Extended squaraine dyes with large two-photon absorption cross-sections," J. Am. Chem. Soc. 128(45), 14444-14445 (2006).

48. J. M. Hales, J. Matichak, S. Barlow, S. Ohira, K. Yesudas, J.-L. Brédas, J. W. Perry, and S. R. Marder, "Design of polymethine dyes with large third-order optical nonlinearities and loss figures of merit," Science 327(5972), 1485-1488 (2010).

49. N. S. Makarov, E. Beuerman, M. Drobizhev, J. Starkey, and A. Rebane, "Environment-sensitive two-photon dye," Proc. SPIE 7049, 70490Y (2008).

50. M. Faraggi, P. Peretz, I. Rosenthal, and D. Weinraub, "Solution properties of dye lasers. Rhodamine B in alcohols," Chem. Phys. Lett. 103(4), 310-314 (1984).

\section{Introduction}

Careful characterization of the two-photon absorption (2PA) properties of organic molecular systems is essential for 2PA applications, such as 3D optical memory [1-5], photodynamic therapy [6-12], optical power limiting [13-19], 2PA microscopy [20-25], nanofabrication [26-32], and others. While providing critical and useful data, absolute measurement of 2PA cross sections ( $\sigma_{2}$ or $\delta$, typically reported in GM where $1 \mathrm{GM}=1 \times 10^{-50} \mathrm{~cm}^{4} \cdot \mathrm{sec} \cdot \mathrm{phot}^{-1}$ ) over a broad range of wavelengths is usually a tedious and time-consuming task because it requires accurate characterization of the excitation beam's energy and spatio-temporal characteristics [33]. Furthermore, if two-photon-excited fluorescence (2PEF) is being monitored, other experimental factors must also be well-characterized, such as the optical collection efficiency and wavelength dependence of the detector sensitivity. This task was significantly simplified following the first publications of reference standards for relative 2PEF measurements [34,35]. Reference compounds with well-characterized 2PA spectra allow one to determine the unknown 2PA cross sections of a solute, by comparing their respective $2 \mathrm{PEF}$ signal intensities. By measuring the sample and reference serially using the same geometry, i.e. single arm [36], the particular aspects of the excitation beam (energy, spatial/temporal profile) and the collection geometry are common to each measurement and therefore need not be accurately characterized. However, fluctuations in the beam characteristics during these sequential acquisitions can cause additional errors. Conversely, measuring the sample and reference simultaneously and normalizing for shot-to-shot intensity variation, i.e. by using a two arm system [37,38], automatically corrects for the variations of the excitation pulse intensity and reduces the overall acquisition time. Previously, care had to be taken in such measurements to ensure that the two arms have nearly identical excitation and collection geometries to properly eliminate their influence. 
Normalization for shot-to-shot intensity variations does not address issues associated with variations in spectral responsivity of diffraction gratings and detectors. However, if the reference compound is chosen such that its fluorescence emission spectrum overlaps with that of the molecule under study, the monitoring of a common narrowband portion of these spectra eliminates the need to account for spectral sensitivity [36,39]. The ability to perform such measurements is, of course, contingent on the existence of reference standards with appropriate emission spectra and reasonable 2PA cross sections. Recently, a large set of reference compounds with excitation wavelengths covering the range 550-1600 nm, and emission wavelengths in the 400-800 $\mathrm{nm}$ spectral range has been reported [33]. However, since this publication, certain reference standards with emission in the $550-800 \mathrm{~nm}$ spectral range have become unavailable and others possess extremely small 2PA cross sections. In particular, zinc tetrakis-(phenylthio)phthalocyanine is no longer available from SigmaAldrich, and tetraphenylporphine has 2PA cross sections below $10 \mathrm{GM}$ and fluorescence quantum yield below $6 \%$. Therefore, better $2 \mathrm{PA}$ standards for this spectral emission range are desired.

In this paper, we present a referential two-channel 2PA technique that is insensitive to source characteristics and instabilities, differences in excitation and collection geometries, and variations in detector response. This compact apparatus allows for rapid and broadband acquisition of 2PA spectra with good accuracy. We have employed this system for characterization of six commercially available dye compounds: Rhodamine-640, Rhodamine700, LDS-698, LDS-798, LDS-925, and Styryl-15 (see Fig. 1 for structures), whose emission covers a critical range of wavelengths, 550-1000 nm. These compounds were found to show reasonably large 2PA cross sections and emission yields, and therefore can be readily used as secondary reference standards for $2 \mathrm{PEF}$ measurements. Furthermore, the determined cross sections were confirmed using the Z-scan nonlinear characterization technique, which is essentially an absolute determination method.

\section{Experimental}

Rhodamine 6G (590 chloride) (CAS\# 3068-39-1), Rhodamine B (610 chloride) (CAS\# 81-889), Rhodamine-640 (CAS\# 72102-91-1), Rhodamine-700 (CAS\# 63561-42-2), LDS-698 (CAS\# 87004-02-2), LDS-798 (CAS\# 92479-59-9), LDS-925 (CAS\# 94507-05-8), Styryl-9M (CAS\# 82988-08-7), and Styryl-15 (CAS\# 385437-35-4) (Exciton Dayton, OH), and spectralquality chloroform (Fisher Scientific, Pittsburg, PA), and methanol (EMD Chemicals Inc., Gibbstown, NJ) were used as obtained without further purification. Solutions with concentrations of $\sim 0.5-2 \mathrm{mM}$ were used for Z-scan, while much lower concentrations, $\sim 0.05$ $\mathrm{mM}$ were used to measure relative 2PA spectra, and $\sim 0.2 \mu \mathrm{M}$ were used for measurements of differential quantum yield for calculation of the 2PA cross sections. Low-concentration solutions of Rhodamine-700 in methanol showed photodecomposition within a few minutes of preparation; however, addition of $0.05 \%$ trifluoroacetic acid prevented this degradation (as pointed out by Exciton technical note).

Our $2 \mathrm{PEF}$ measurement technique is similar to that described previously [33]. Our laser system consists of a Ti:Sapphire regenerative amplifier (Spitfire, Spectra-Physics, 800-nm, 1$\mathrm{W}$ average power, $100-\mathrm{fs}$ pulse width, $1-\mathrm{kHz}$ repetition rate) pumped by a intracavity frequency-doubled Nd:YLF laser (Empower, Spectra-Physics). The amplifier is seeded by a Ti:Sapphire femtosecond oscillator (Tsunami, Spectra-Physics), which is pumped by an intracavity frequency-doubled $\mathrm{Nd}: \mathrm{YVO}_{4}$ laser (Millenia, Spectra-Physics). We utilize pulses from the amplifier to pump a computer-controlled optical parametric amplifier (OPA; TOPAS-C, Light-Conversion) with a unidirectional output. The output of the OPA ( 100 mW average power) is used to excite $2 \mathrm{PA}$-induced fluorescence and can be continuously tuned under computer control over a broad wavelength range, 1100-2800 nm (typical FWHM 10-30 $\mathrm{nm})$. The second harmonic of the OPA output can be used to reach wavelengths in the range of 550-1100 nm ( 30 mW average power).

$\# 146980$ - \$15.00 USD

(C) 2011 OSA
Received 3 May 2011; revised 29 Jun 2011; accepted 30 Jun 2011; published 7 Jul 2011 1 August 2011 / Vol. 1, No. 4 / OPTICAL MATERIALS EXPRESS 554 


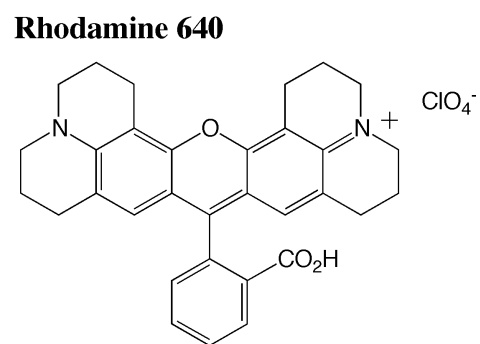

LDS-698

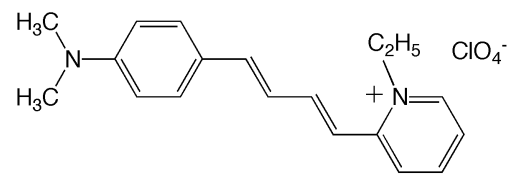

Rhodamine 700

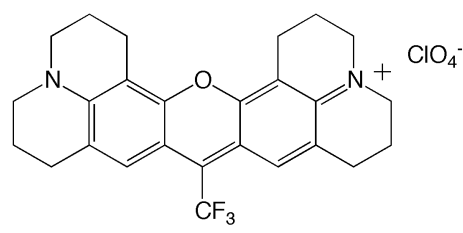

LDS-798

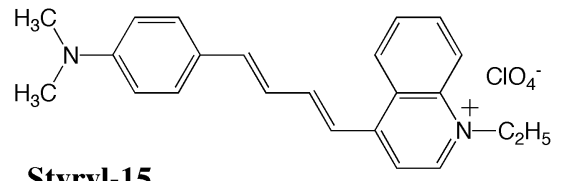

Styryl-15

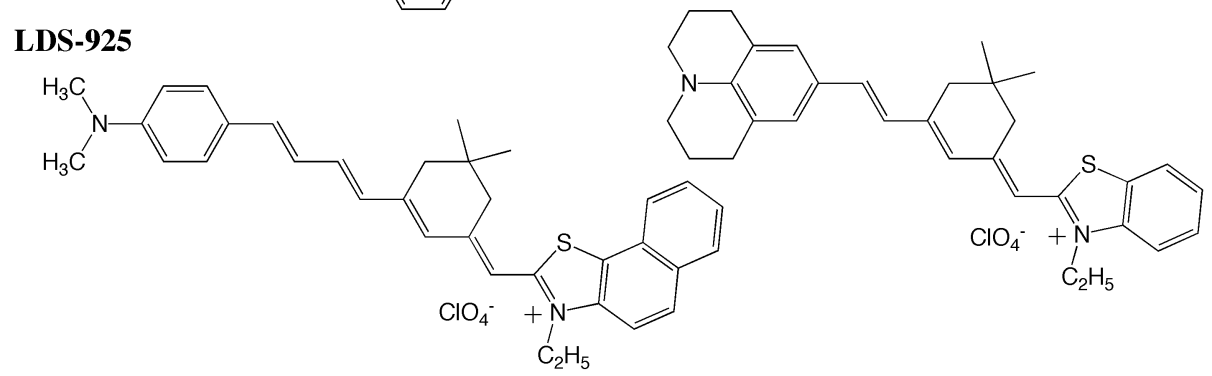

Fig. 1. Chemical structures of six commercially available laser dyes measured.

Our experimental setup is shown in Fig. 2. The excitation beam out of the OPA first passes through a set of neutral density (ND) filters mounted on two filter wheels - one is a continuous ND filter to control the excitation power during power dependence and cross section measurements, and the other is a set of fixed 1 inch diameter ND filters to provide roughly the same excitation power across various ranges of the OPA output. A small portion of the femtosecond pulsed laser beam is sampled and is directed into a monochromator (MicroHR, Horiba), the output of which is sent to a sandwich diode photo-detector (DSD2, ThorLabs) to monitor the OPA output wavelength during the 2PEF measurements and the beam intensity during measurements of the dependence of the fluorescence on excitation intensity. The remaining portion of the beam is weakly focused between the two samples (S1 and S2) with a $125 \mathrm{~mm}$ focal length lens L1. Potential cross-talk from emission of the two samples is prevented by using a pinhole aperture (much larger than the beam size) as a block (SB) placed between the samples. The excitation beam is blocked after the samples with a beam block (BB). The average excitation power incident on the samples during the measurements of 2PA spectra is kept between $\sim 0.2-5 \mathrm{~mW}$, while the laser pulse duration is 75-150 fs (FWHM), and the spot size on the sample is $\sim 0.2 \mathrm{~mm}$ (HW1/eM). In choosing the appropriate laser power, consideration should be given to avoiding white-light generation, depletion of the ground state population [40], and thermal and nonlinear refraction effects. The emission from the samples is collected by aspheric lenses L2 and L3 (focal length $=8$ $\mathrm{mm}$; KPA016-C, Newport) and is sent through a set of short pass filters mounted in rotating wheels (SPFW1 and SPFW2). The collimated fluorescence is collected by fiber collimators (F810SMA-543, ThorLabs) and sent through a two-arm optical fiber bundle (BFB-455-7, Princeton Instruments) to an imaging fiber adapter (FC-446-030, Princeton Instruments) that is coupled to a monochromator (SpectraPro-150, Acton). The delivery/fiber collection system affords great flexibility in the system design. The fluorescence spectra of the sample and the reference are detected separately as two regions of interest on a CCD camera (LN/CCD1100PB, Roper Scientific, controller: ST-133, Roper Scientific). Simultaneous detection of the spectra coupled with proper binning of the CCD can provide quite rapid acquisition. It

\#146980 - \$15.00 USD

(C) 2011 OSA 
should be noted that the footprint for the excitation-sample-collection geometry is quite small $(250 \mathrm{~mm} \times 150 \mathrm{~mm})$ while the entire experimental arrangement shown in Fig. 2 also occupies a reasonably small area, $\sim 80 \mathrm{~cm} \times 80 \mathrm{~cm}$. The acquisition time is kept in the range of $\sim 0.5-5$ seconds per step, but could be increased up to $\sim 120$ seconds for low-fluorescence samples. Further increase of the exposures is prohibited by the increasing dark electron noise in the CCD signal, which is usually kept $<10^{-2}$ of the fluorescence signal. Under these conditions we can measure 2PA cross sections with brightness (product of fluorescence quantum yield and 2PA cross sections) as low as $\sim 10^{-3} \mathrm{GM}$, and relative 2PA spectra with brightness as low as $\sim 10^{-5} \mathrm{GM}$.

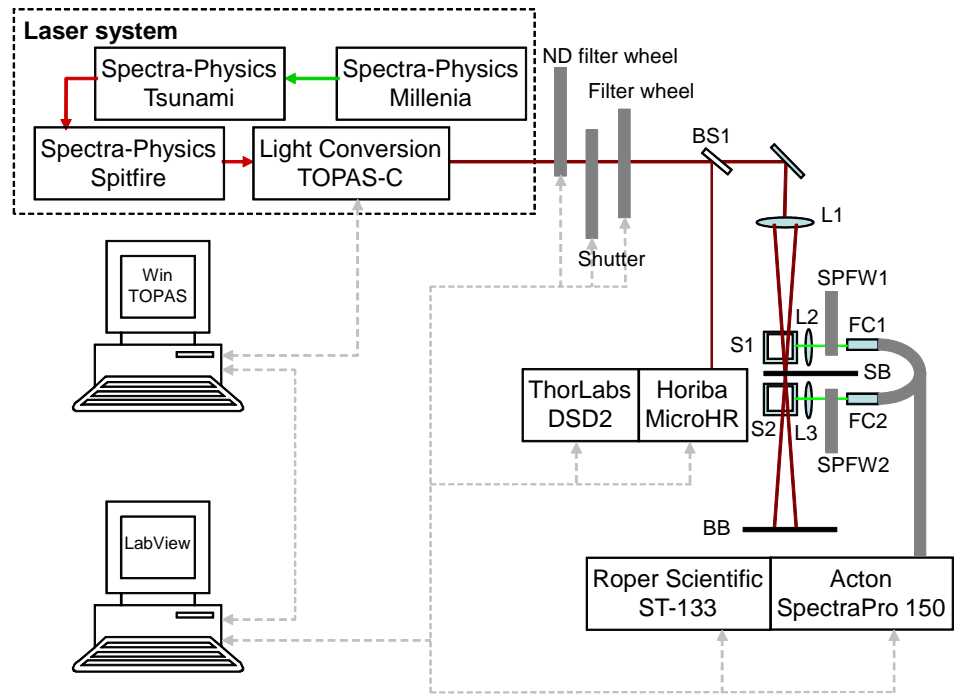

Fig. 2. Schematic of the dual-channel two-photon-induced fluorescence experimental setup. BS1 is the beam splitter; L1 is the focusing lens $(\mathrm{f}=125 \mathrm{~mm})$; S1 and S2 are the sample holders, L2 and L3 are the collection lenses ( $\mathrm{f}=8 \mathrm{~mm}$ ); SPFW-1 and SPFW-2 are the filter wheels with the short pass filters to cut-off the laser scattering; FC1 and FC2 are the fiber collimation lenses; SB is the aperture used to block laser scattering and potential fluorescence cross talk between the two channels; BB is the beam block.

The measurements were performed by scanning the laser wavelength and simultaneously collecting the 2PEF signals from the two channels, effectively a "two-arm" technique as described above. It should be noted that no care was taken to ensure the two arms have identical excitation/collection geometries, however this will not be of consequence as described below. For each spectral range, two sets of measurements were undertaken: the sample is first placed in channel one, while the reference is in channel two, and then the measurement is repeated with the sample and reference exchanged. Under these conditions the time-averaged fluorescence signals, for the sample or reference, integrated over the exposure time $T$ are:

$$
F\left(\lambda_{e x}\right)=\frac{\sigma_{2}\left(\lambda_{e x}\right) C I_{0}^{2}\left(\lambda_{e x}\right)}{2(h v)^{2}} \int_{-\infty}^{\infty} \int_{-\infty}^{\infty} \int_{-\infty}^{\infty} \bar{I}^{2}\left(x, y, z, \lambda_{e x}\right) d x d y d z \int_{0}^{T} \bar{I}^{2}\left(t, \lambda_{e x}\right) d t \int_{\lambda_{\min }}^{\lambda_{\max }} \eta\left(\lambda^{\prime}\right) \phi\left(\lambda^{\prime}\right) d \lambda^{\prime},(1)
$$

where $\sigma_{2}\left(\lambda_{e x}\right)$ is the $2 \mathrm{PA}$ cross section at the excitation wavelength, $C$ is the concentration (in $\mathrm{cm}^{-3}$ ), $I_{0}$ is the peak irradiance in the sample, $h$ is Planck's constant, $v$ is the excitation frequency, $\bar{I}\left(x, y, z, \lambda_{e x}\right)$ is the normalized spatial beam profile, $\bar{I}\left(t, \lambda_{e x}\right)$ is the normalized temporal beam profile, $\eta(\lambda)$ is the collection efficiency, $\varphi(\lambda)$ is the differential quantum 
yield, and $\lambda_{\min }$ and $\lambda_{\max }$ are the minimum and maximum wavelengths over which the emission spectra of the compounds are integrated.

The 2PA cross section of the sample when measured in channels one and two, respectively, (i.e. when the positions of the reference and sample under study are reversed) can then be expressed as:

$$
\begin{aligned}
& \sigma_{2}^{S, 1}\left(\lambda_{e x}\right)=\sigma_{2}^{R}\left(\lambda_{e x}\right) \frac{C^{R}}{C^{S}} \frac{I_{0(2)}^{2(R)}\left(\lambda_{e x}\right)}{I_{0(1)}^{2(S)}\left(\lambda_{e x}\right)} \frac{F_{1}^{S}\left(\lambda_{e x}\right)}{F_{2}^{R}\left(\lambda_{e x}\right)} \frac{\iint_{-\infty}^{\infty} \int_{-\infty-\infty}^{\infty} \int_{-\infty}^{\infty} \int_{-\infty}^{\infty} \int_{-\infty}^{\infty} \bar{I}_{1}^{2(S)}\left(x, y, z, \lambda_{e x}\right) d x d y d z}{\left.x, \lambda_{e x}\right) d x d y d z} \times \\
& \frac{\int_{0}^{T} \bar{I}_{2}^{2(R)}\left(t, \lambda_{e x}\right) d t \int_{\lambda_{\min }^{R}}^{\lambda_{\max }^{R}} \eta_{2}\left(\lambda^{\prime}\right) \phi^{R}\left(\lambda^{\prime}\right) d \lambda^{\prime}}{\int_{0}^{T} \bar{I}_{1}^{2(S)}\left(t, \lambda_{e x}\right) d t \int_{\lambda_{\min }^{S}}^{\frac{\lambda_{\min }^{S}}{\lambda_{\text {nax }}^{x}}} \eta_{1}\left(\lambda^{\prime}\right) \phi^{S}\left(\lambda^{\prime}\right) d \lambda^{\prime}} \\
& \sigma_{2}^{S, 2}\left(\lambda_{e x}\right)=\sigma_{2}^{R}\left(\lambda_{e x}\right) \frac{C^{R}}{C^{S}} \frac{I_{0(1)}^{2(R)}\left(\lambda_{e x}\right)}{I_{0(2)}^{2(S)}\left(\lambda_{e x}\right)} \frac{F_{2}^{S}\left(\lambda_{e x}\right)}{F_{1}^{R}\left(\lambda_{e x}\right)} \frac{\iint_{-\infty-\infty}^{\infty} \int_{-\infty}^{\infty} \int_{-\infty}^{\infty} \int_{-\infty}^{\infty} \bar{I}_{1}^{2(R)}\left(x, y, z, \lambda_{e x}\right) d x d y d z}{I_{2}^{2(S)}\left(x, y, z, \lambda_{e x}\right) d x d y d z} \times
\end{aligned}
$$

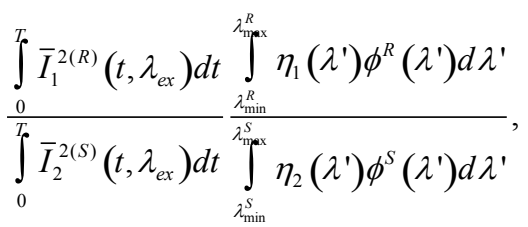

where superscripts $\mathrm{R}$ and $\mathrm{S}$ stand for reference and sample respectively, while subscripts 1 and 2 indicate channels one and two. Assuming that the beam depletion is negligible, one can show that:

$$
\begin{aligned}
& \frac{I_{0(2)}^{2(R)}\left(\lambda_{e x}\right)}{I_{0(1)}^{2(S)}\left(\lambda_{e x}\right)} \frac{I_{0(1)}^{2(R)}\left(\lambda_{e x}\right)}{I_{0(2)}^{2(S)}\left(\lambda_{e x}\right)}=1 ; \frac{\int_{0}^{T} \bar{I}_{2}^{2(R)}\left(t, \lambda_{e x}\right) d t}{\int_{0}^{T} \bar{I}_{1}^{2(S)}\left(t, \lambda_{e x}\right) d t} \frac{\bar{I}_{1}^{2(R)}\left(t, \lambda_{e x}\right) d t}{\int_{0}^{T} \bar{I}_{2}^{2(S)}\left(t, \lambda_{e x}\right) d t}=1 \\
& \int_{-\infty-\infty}^{\infty} \int_{-\infty}^{\infty} \bar{I}_{2}^{2(R)}\left(x, y, z, \lambda_{e x}\right) d x d y d z \iint_{-\infty-\infty}^{\infty} \int_{-\infty}^{\infty} \bar{I}_{1}^{2(R)}\left(x, y, z, \lambda_{e x}\right) d x d y d z \\
& \iint_{-\infty}^{\infty} \int_{-\infty}^{\infty} \int_{-\infty}^{\infty} \bar{I}_{1}^{2(S)}\left(x, y, z, \lambda_{e x}\right) d x d y d z \iint_{-\infty}^{\infty} \int_{-\infty}^{\infty} \int_{-\infty}^{\infty} \bar{I}_{2}^{2(S)}\left(x, y, z, \lambda_{e x}\right) d x d y d z
\end{aligned}
$$

Therefore,

$$
\begin{aligned}
& \sigma_{2}^{S}\left(\lambda_{e x}\right)=\sqrt{\sigma_{2}^{S, 1}\left(\lambda_{e x}\right) \sigma_{2}^{S, 2}\left(\lambda_{e x}\right)}=
\end{aligned}
$$

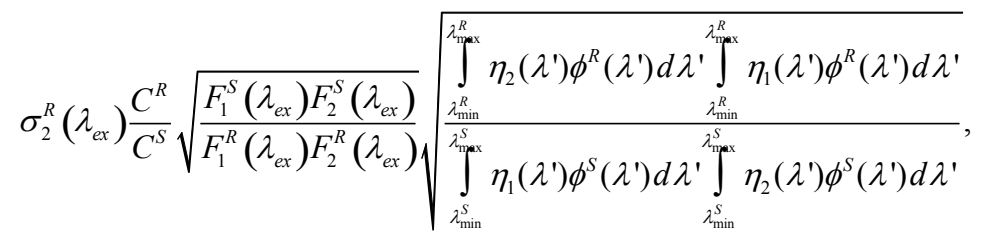

where

\#146980 - \$15.00 USD

(C) 2011 OSA 


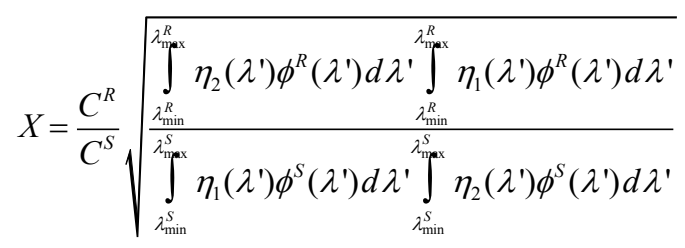

is just a constant, which does not depend on the excitation wavelength. Note also that any effects on the irradiance distribution in the sample and reference cells due to different refractive indices in expression (3) are corrected for by measurements with the positions of the sample and reference exchanged.

One can therefore obtain the relative $2 \mathrm{PA}$ spectrum as:

$$
S\left(\lambda_{e x}\right)=\sigma_{2}^{R}\left(\lambda_{e x}\right) \sqrt{\frac{F_{1}^{S}\left(\lambda_{e x}\right) F_{2}^{S}\left(\lambda_{e x}\right)}{F_{1}^{R}\left(\lambda_{e x}\right) F_{2}^{R}\left(\lambda_{e x}\right)}}
$$

and subsequently normalize the spectrum to the $2 \mathrm{PA}$ cross section, measured at a single wavelength, $\lambda_{0}$, as:

$$
\sigma_{2}^{S}\left(\lambda_{e x}\right)=S\left(\lambda_{e x}\right) \frac{\sigma_{2}^{S}\left(\lambda_{0}\right)}{S\left(\lambda_{0}\right)} .
$$

Various concentrations of the samples can be used for the measurements of the relative 2PA spectra [Eq. (6)], regardless of reabsorption of fluorescence since this will be same for all excitation wavelengths. The cross section at a single wavelength can be obtained without taking into account spectral sensitivity of the fluorescence detection if the integration across the fluorescence wavelengths in expression (4) is replaced by a narrowband ( 1-nm) value of the differential quantum yield at a common emission wavelength $\lambda^{\prime}$ for sample and reference $\left(\lambda^{\prime}=\lambda_{\min }^{S, R}=\lambda_{\max }^{S, R}\right.$ ), chosen such that there is no reabsorption of fluorescence in both arms at this particular wavelength, as:

$$
\sigma_{2}^{S}\left(\lambda_{0}\right)=\sigma_{2}^{R}\left(\lambda_{0}\right) \frac{C^{R}}{C^{S}} \sqrt{\frac{F_{1}^{S}\left(\lambda_{0}\right) F_{2}^{S}\left(\lambda_{0}\right)}{F_{1}^{R}\left(\lambda_{0}\right) F_{2}^{R}\left(\lambda_{0}\right)}} \frac{\phi^{R}\left(\lambda^{\prime}\right)}{\phi^{S}\left(\lambda^{\prime}\right)} .
$$

It therefore becomes clear that the acquired 2PA cross section is not dependent on any difference in excitation or collection geometry between the two arms, nor any differences in detector efficiency. Furthermore, the ratio,

$$
\frac{\phi^{R}\left(\lambda^{\prime}\right)}{\phi^{S}\left(\lambda^{\prime}\right)}=\frac{f^{R}\left(\lambda^{\prime}\right)}{1-10^{-O D_{I P A}^{R}}} \frac{1-10^{-O D_{1 P A}^{S}}}{f^{S}\left(\lambda^{\prime}\right)}
$$

where $f\left(\lambda^{\prime}\right)$ stands for the fluorescence signal at a specific wavelength $\lambda^{\prime}$, excited in lowconcentration solutions $\left(O D_{1 P A}^{R, S}<0.1\right)$ via one-photon excitation, can be obtained with a regular spectrofluorimeter, as discussed in [33], or could be obtained in the current 2PEF apparatus by simply generating one-photon induced fluorescence. Note that the $n^{2}$ dependence of the emission collection efficiency on the refractive index of the sample and reference is identical for two- and one-photon excitation, and therefore cancels out in the final expression for the cross section.

Since the measurements are not sensitive to pulse-to-pulse instabilities of the laser, if the experimental conditions can be chosen such that the fluorescence signals are significantly stronger than the detection shot noise, the accuracy of the measured spectra is essentially the same as for the applied reference standards (i.e. $\pm 15 \%$ ). However, it should be noted that all 
fluorescence signals were verified to be within the regime of quadratic dependence on the incident irradiance.

All aspects of the measurement process, including the wavelength scan with arbitrary steps, data acquisition, and correction of the relative spectra using references are automated and computer controlled using LabView. This automation coupled with the simultaneous detection of reference and sample spectra allows for rapid acquisition. For instance, a typical acquisition (including both sets of measurements) over an excitation range of $\sim 200 \mathrm{~nm}$ can be accomplished in the span of less than 10 minutes.

In the experiments discussed here, we measured 2PA spectra relative to Rhodamine $\mathrm{B}$ dissolved in methanol, Rhodamine 6G dissolved in methanol, and Styryl-9M dissolved in chloroform [33], using the values at wavelengths $\lambda_{0}=820 \mathrm{~nm}, \lambda_{0}=930 \mathrm{~nm}, \lambda_{0}=1000 \mathrm{~nm}$, and $\lambda_{0}=1200 \mathrm{~nm}$ to calibrate the absolute $2 \mathrm{PA}$ cross sections. We also performed femtosecond-pulsed open-aperture Z-scan experiments for LDS-698 and LDS-798 at selected wavelengths (spanning from $900 \mathrm{~nm}$ to $1350 \mathrm{~nm}$ in $50 \mathrm{~nm}$ steps) to verify the accuracy of the $2 \mathrm{PEF}$ method. The experimental setup and procedure used for Z-scan has been described previously [41,42]. The excitation pulse energies varied from $50 \mathrm{~nJ}$ to $1.2 \mu \mathrm{J}$, the Raleigh range from 3 to $6 \mathrm{~mm}$, the M-value from 1.01 to 1.07 , and the pulse duration from 60 to 120 fs. The experimental error of the Z-scan measurements $( \pm 30 \%)$ consists of $\sim 20 \%$ error in the determination of the laser pulse duration, $\sim 10 \%$ error in the determination of the spatial dimensions of the laser beam (in $\mathrm{x}$ - and $\mathrm{y}$ - direction), $\sim 10 \%$ error in the fit of experimental nonlinear transmission data, $\sim 5 \%$ error in the concentration of the high-concentrated solutions, and finally $\sim 5 \%$ error in the excitation laser power. The laser pulse durations were determined using semiconductor references with known nonlinearities (fused silica and zinc sulfide) instead of the autocorrelation measurements, and therefore yield relatively large errors.

\section{Results and Discussion}

Figure 3 presents the molar extinction coefficients, normalized fluorescence and 2PA spectra of the compounds plotted versus 2PA laser wavelength (bottom scale), which is twice the onephoton absorption (1PA) transition wavelength (top horizontal scale). The insets show the names of the compounds, and the corresponding solvent that was used, while the black squares show 2PA cross sections obtained from the Z-scan experiments. A tabulated version of the 2PA spectra from the fluorescence excitation method is also given in Table 1.

The 1PA and 2PA spectra of the Rhodamines are similar to those measured previously for other types of Rhodamines [33]. The spectra of Rhodamine-640 and Rhodamine-700 are both red-shifted compared to Rhodamine B. While in 1PA the lowest energy transition is the strongest, and is followed by a weaker vibronic side-band, an opposite behavior is observed in 2PA. There, the intensity of the vibronic peak is stronger, while the $0-0$ transition manifests itself as a shoulder; this effect is qualitatively similar to many other compounds explored previously $[21,43,44]$. Similar effects were also observed for many $D-\pi$-D and D-A-D types of compounds [45,46], as well as squarines [47] and cyanines [48], which have symmetry or effective symmetry due to high electron delocalization. While the shorter wavelength 1PA transitions are relatively weak in these Rhodamines, 2PA transitions in the same spectral region exhibit a rather strong response. This is consistent with a reasonably high degree of symmetry in these molecules leading to drastically different dipole-selection rules for 1PA and $2 \mathrm{PA}$ activity. 

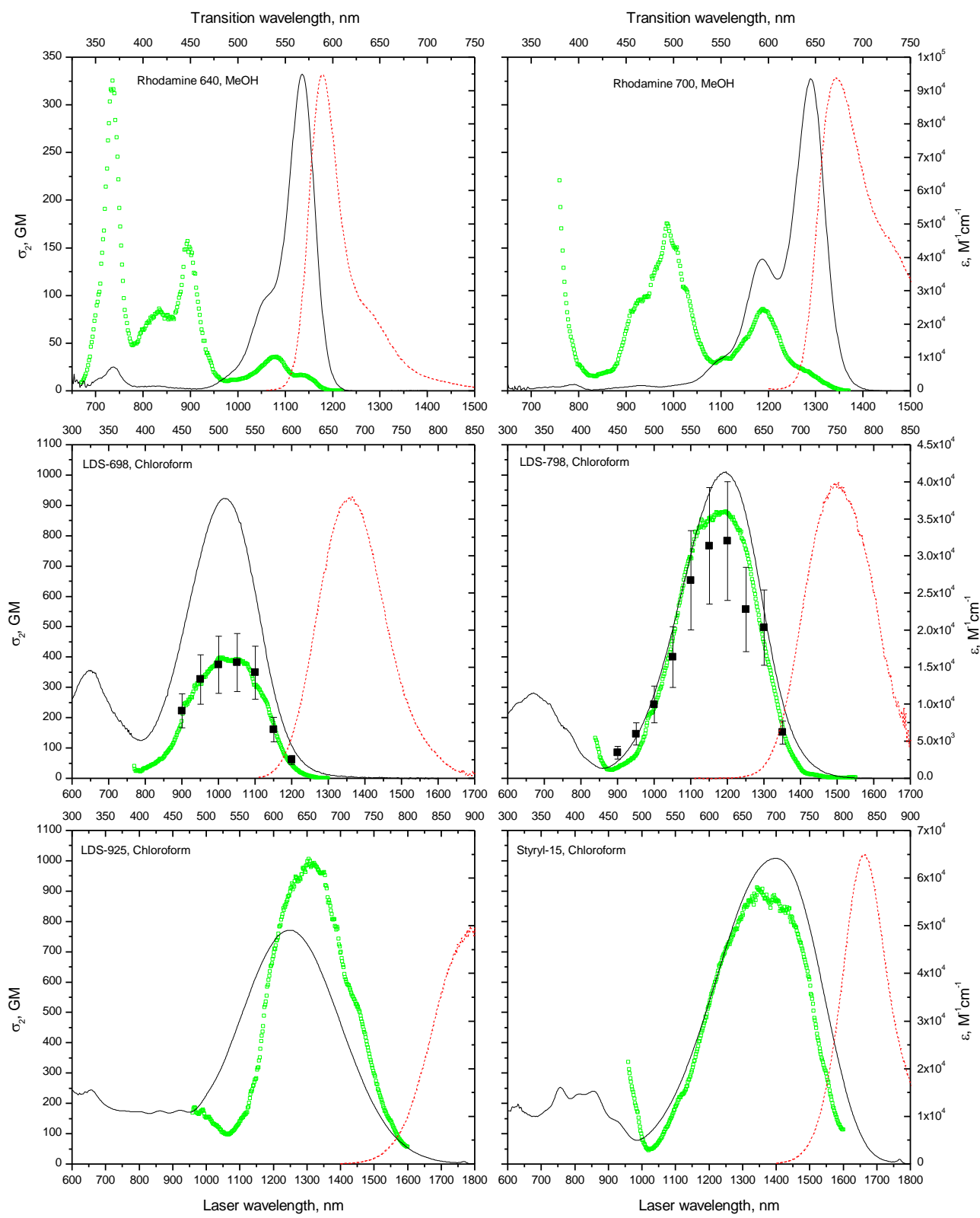

Fig. 3. 1PA (black solid line, right vertical and top horizontal scales), normalized fluorescence (red dash-dot line, top horizontal scale), and 2PA (green symbols, left and bottom scales) spectra of the compounds. Black squares show the $2 \mathrm{PA}$ cross sections obtained with the Z-scan experiments. 2PA cross sections are given in GM where $1 \mathrm{GM}=1 \times 10^{-50} \mathrm{~cm}^{4} \cdot \mathrm{sec} \cdot \mathrm{phot}^{-1}$.

For the LDS/Styryl compounds, both the 1PA and 2PA spectra show significant activity into the same broad low-energy transition. The lack of molecular symmetry and dipolar charge transfer in these systems leads to states with no definite parity and therefore 1PA and 2PA processes are able to access the same electronic states. The 1PA spectra also show another weaker transition at shorter wavelengths, which is not accessible by 2PA measurements due to 1PA absorption in the low energy portion of the linear absorption spectrum. The spectra are qualitatively similar to that of Styryl-9M $[33,49]$. They are blueshifted relative to Styryl-9M in case of LDS-698 and LDS-798 due to shorter conjugation 
length. The 1PA and 2PA peaks of Styryl-15 and LDS-925 are red-shifted relative to Styryl$9 \mathrm{M}$, which can be attributed to the presence of the more planarized julolidine group of Styryl15, relative to the dialkyl amine donor of Styryl-9M, and longer conjugation of LDS-925.

Table 1. 2PA Cross Sections (given in GM where $1 \mathrm{GM}=1 \times 10^{-50} \mathrm{~cm}^{4} \cdot \sec \cdot \mathrm{phot}^{-1}$ ) of the Compounds at Selected Wavelengths, Determined by Fluorescence-Based 2PA

Measurements Relative to Compounds from [33] ${ }^{a, b}$

\begin{tabular}{|c|c|c|c|c|c|c|}
\hline Wavelength, $\mathrm{nm}$ & Rhodamine 640 & Rhodamine 700 & LDS-698 & LDS-798 & LDS-925 & Styryl-15 \\
\hline 670 & 8.6 & & & & & \\
\hline 680 & 18 & & & & & \\
\hline 690 & 46 & & & & & \\
\hline 700 & 79 & & & & & \\
\hline 710 & 110 & & & & & \\
\hline 720 & 190 & & & & & \\
\hline 730 & 305 & & & & & \\
\hline 740 & 310 & & & & & \\
\hline 750 & 190 & & & & & \\
\hline 760 & 97 & 220 & & & & \\
\hline 770 & 60 & 115 & 40 & & & \\
\hline 780 & 48 & 68 & 26 & & & \\
\hline 790 & 52 & 43 & 26 & & & \\
\hline 800 & 64 & 28 & 33 & & & \\
\hline 810 & 71 & 21 & 38 & & & \\
\hline 820 & 78 & 17 & 45 & & & \\
\hline 830 & 82 & 16 & 52 & & & \\
\hline 840 & 82 & 16 & 63 & 130 & & \\
\hline 850 & 79 & 18 & 68 & 85 & & \\
\hline 860 & 78 & 19 & 86 & 50 & & \\
\hline 870 & 83 & 21 & 100 & 33 & & \\
\hline 880 & 105 & 28 & 125 & 28 & & \\
\hline 890 & 150 & 44 & 155 & 30 & & \\
\hline 900 & 150 & 63 & 190 & 34 & & \\
\hline 910 & 125 & 84 & 250 & 42 & & \\
\hline 920 & 80 & 89 & 255 & 48 & & \\
\hline 930 & 52 & 96 & 280 & 56 & & \\
\hline 940 & 34 & 99 & 300 & 66 & & \\
\hline 950 & 20 & 100 & 310 & 75 & & \\
\hline 960 & 14 & 125 & 335 & 98 & 170 & 335 \\
\hline 970 & 11 & 135 & 350 & 125 & 170 & 255 \\
\hline 980 & 9.9 & 155 & 360 & 175 & 165 & 200 \\
\hline 990 & 11 & 170 & 370 & 210 & 180 & 155 \\
\hline 1000 & 12 & 150 & 395 & 230 & 155 & 89 \\
\hline 1010 & 13 & 145 & 395 & 265 & 155 & 63 \\
\hline 1020 & 14 & 110 & 390 & 300 & 130 & 45 \\
\hline 1030 & 17 & 105 & 390 & 355 & 125 & 50 \\
\hline 1040 & 20 & 79 & 390 & 395 & 115 & 58 \\
\hline 1050 & 25 & 59 & 390 & 450 & 105 & 71 \\
\hline 1060 & 30 & 46 & 390 & 515 & 98 & 88 \\
\hline 1070 & 34 & 35 & 380 & 580 & 99 & 115 \\
\hline 1080 & 35 & 31 & 375 & 635 & 110 & 130 \\
\hline 1090 & 33 & 29 & 345 & 700 & 125 & 160 \\
\hline 1100 & 26 & 33 & 305 & 730 & 140 & 190 \\
\hline 1110 & 19 & 34 & 295 & 790 & 155 & 210 \\
\hline 1120 & 16 & 33 & 270 & 810 & 170 & 230 \\
\hline 1130 & 16 & 39 & 255 & 855 & 215 & 245 \\
\hline 1140 & 16 & 46 & 215 & 835 & 255 & 280 \\
\hline 1150 & 14 & 53 & 180 & 860 & 280 & 310 \\
\hline 1160 & 11 & 59 & 140 & 850 & 350 & 350 \\
\hline 1170 & 6.0 & 70 & 110 & 880 & 455 & 405 \\
\hline 1180 & 2.6 & 82 & 80 & 870 & 555 & 440 \\
\hline 1190 & 0.78 & 85 & 60 & 880 & 635 & 485 \\
\hline 1200 & 0.16 & 82 & 46 & 875 & 685 & 525 \\
\hline 1210 & 0.049 & 72 & 33 & 860 & 735 & 565 \\
\hline
\end{tabular}

\#146980 - \$15.00 USD

(C) 2011 OSA
Received 3 May 2011; revised 29 Jun 2011; accepted 30 Jun 2011; published 7 Jul 2011

1 August 2011 / Vol. 1, No. 4 / OPTICAL MATERIALS EXPRESS 561 


\begin{tabular}{|c|c|c|c|c|c|c|}
\hline 1220 & 0.037 & 60 & 23 & 845 & 785 & 600 \\
\hline 1230 & & 46 & 16 & 820 & 845 & 655 \\
\hline 1240 & & 36 & 11 & 790 & 865 & 680 \\
\hline 1250 & & 29 & 7.2 & 755 & 900 & 710 \\
\hline 1260 & & 25 & 4.3 & 710 & 920 & 730 \\
\hline 1270 & & 23 & 2.1 & 645 & 940 & 755 \\
\hline 1280 & & 21 & 0.95 & 575 & 940 & 770 \\
\hline 1290 & & 18 & 0.61 & 510 & 975 & 800 \\
\hline 1300 & & 15 & 0.49 & 450 & 985 & 825 \\
\hline 1310 & & 11 & & 390 & 1000 & 850 \\
\hline 1320 & & 8.1 & & 345 & 985 & 870 \\
\hline 1330 & & 5.1 & & 290 & 980 & 875 \\
\hline 1340 & & 2.9 & & 230 & 960 & 880 \\
\hline 1350 & & 1.3 & & 175 & 960 & 900 \\
\hline 1360 & & 0.24 & & 130 & 910 & 910 \\
\hline 1370 & & 0.0035 & & 96 & 850 & 880 \\
\hline 1380 & & & & 73 & 805 & 870 \\
\hline 1390 & & & & 53 & 780 & 870 \\
\hline 1400 & & & & 34 & 695 & 870 \\
\hline 1410 & & & & 20 & 650 & 860 \\
\hline 1420 & & & & 14 & 595 & 835 \\
\hline 1430 & & & & 13 & 575 & 835 \\
\hline 1440 & & & & 11 & 545 & 840 \\
\hline 1450 & & & & 8.9 & 520 & 800 \\
\hline 1460 & & & & 6.1 & 490 & 800 \\
\hline 1470 & & & & 4.1 & 440 & 740 \\
\hline 1480 & & & & 3.0 & 395 & 705 \\
\hline 1490 & & & & 2.5 & 345 & 655 \\
\hline 1500 & & & & 2.2 & 300 & 610 \\
\hline 1510 & & & & 1.9 & 260 & 530 \\
\hline 1520 & & & & 1.9 & 220 & 450 \\
\hline 1530 & & & & 2.1 & 185 & 380 \\
\hline 1540 & & & & 2.4 & 160 & 335 \\
\hline 1550 & & & & 2.9 & 145 & 300 \\
\hline 1560 & & & & & 120 & 240 \\
\hline 1570 & & & & & 95 & 190 \\
\hline 1580 & & & & & 76 & 155 \\
\hline 1590 & & & & & 63 & 130 \\
\hline 1600 & & & & & 57 & 110 \\
\hline
\end{tabular}

${ }^{a}$ The relative errors are estimated to be $\pm 15 \%$.

${ }^{b}$ Even though the cross sections are shown here in 10-nm steps, the data shown in Fig. 3 were measured with 2-nm steps. Although the bandwidth of the OPA pulses was $\sim 10 \mathrm{~nm}$, the effective spectral resolution was $\sim 7 \mathrm{~nm}$ due to the quadratic intensity dependence of the 2PA. Nonetheless, smaller steps are used in our measurements to address two issues: first, for peak widths somewhat smaller or larger than the bandwidth of the pulses, the use of smaller steps allows to obtain more information about the line shapes, as well as more accurately to define the position of each peak. Second, the use of smaller steps allows the identification of systematic errors at individual wavelengths, for example, due to artifacts in optical frequency generation, and distinguishing them from real data associated with narrow peaks. The excitation wavelength for each pulse was determined by our wavelength measurement channel to an accuracy within $\pm 1 \mathrm{~nm}$, and, therefore, we used 2-nm steps for all measurements.

The Z-scan results show good agreement with the fluorescence-based measurements at nearly every excitation wavelength, within the experimental error. The agreement is best for LDS-698, and only slightly worse for LDS-798. The LDS-798 data point at $\lambda=1250 \mathrm{~nm}$ shows the worst agreement between the Z-scan and fluorescence-based measurements. This might be due to the quality of the laser beam, which was found to be poorest at this wavelength $(M=1.07)$. The agreement is also poorer towards shorter wavelengths where we obtained power-dependent 2PA coefficients from the Z-scan measurements (at $\lambda \leq 950 \mathrm{~nm}$ for LDS-698 and at $\lambda \leq 1050 \mathrm{~nm}$ for LDS-798). This is most likely due to the occurrence of additional optical absorption effects, such as excited state absorption. In these cases, the 2PA cross sections were estimated based on a linear extrapolation to zero excitation power.

A few specific notes should be given regarding usage of reference standards in general as well as the specific compounds discussed here. When using the data reported herein as 
secondary reference standards for $2 \mathrm{PEF}$ measurements, it is important to dissolve the standards in the same solvent reported. While Rhodamines show small solvatochromic shifts between various solvents, their transitions are sharp, and therefore the usage of a different solvent could lead to serious errors in the $2 \mathrm{PEF}$ measurements, especially on the slopes of the transition peaks. LDS/Styryl dyes on the other hand are found to have broad transitions, but due to their large permanent dipole moment differences between the ground and excited states [49] they show significant solvatochromic effects. Their 2PA strength is also sensitive to the choice of solvent [49], which could lead to even more significant errors in referential measurements if a different solvent is used. It is also known that the 1PA spectrum of Rhodamine $\mathrm{B}$ is sensitive to the effective $\mathrm{pH}$ of the solvent [50], which is manifested in spectral shifts of the 1PA spectrum (up to $7 \mathrm{~nm}$ ) in methanol, depending on the water content. However, the 2PA spectrum does not show such shifts.

We observed 1-2\% changes in the peak linear absorption of Styryl-9M and LDS-925 after 20 minutes of laser irradiation with average excitation powers of about $5 \mathrm{~mW}$. We also observed significant changes in the 1PA of Styryl-9M after a few hours of laser irradiation. Note also that the choice of chloroform for LDS/Styryl dyes was made to avoid significant solvent absorption with near-IR wavelengths $(>1100 \mathrm{~nm})$, where many other solvents usually show appreciable absorption due to $\mathrm{C}-\mathrm{H}$ overtones and combination bands. However, in this solvent it is difficult to obtain concentrations of LDS-698 higher than $0.5 \mathrm{mM}$, which might cause difficulties in Z-scan measurements. In this regard, the $2 \mathrm{PA}$ spectrum of Rhodamine700 was measured in $1-\mathrm{mm}$ cuvette at longer near-IR wavelengths, $\lambda>1150 \mathrm{~nm}$, to ensure no artifacts are present due to the absorption of methanol. We also compared spectra of LDS-925 and Styryl-15 in chloroform and deuterated chloroform around $1410 \mathrm{~nm}$, but did not find a significant difference because of our reference was dissolved in the same solvent. Measurements of the unknown samples in different solvents around this wavelength relative to LDS/Styryl dyes however, require either use of thin 1-mm cuvettes or deuterated chloroform to avoid artifacts due to solvent absorption.

\section{Concluding Remarks}

We have developed a compact, two-channel femtosecond fluorescence excitation apparatus that allows for rapid, high-resolution acquisition of 2PA spectra of solutions. This referential technique is insensitive to source characteristics and instabilities, differences in excitation and collection geometries, and variations in detector response. As a result our instrumental accuracy is mainly limited by the errors associated with the reference standards used. Using well-known reference standards, we measured 2PA spectra of six commercial organic dyes selected to serve as secondary reference standards for fluorescence-based 2PA measurements with emission wavelengths in the spectral range 550-1000 $\mathrm{nm}$. The 2PA spectra of these compounds cover a range of excitation wavelengths from 670 to $1600 \mathrm{~nm}$. The results from this method compare favorably to those determined from a well-known, absolute nonlinear characterization method, femtosecond-pulsed Z-scan.

\section{Acknowledgments}

The work was supported by the DARPA ZOE program (Grant No. W31P4Q-09-1-0012) and the NSF PREM (Grant No. DMR-0934212). We thank Prof. A. Rebane for providing preliminary 2PA data on Rhodamine-640 that were obtained (for $\lambda<1100 \mathrm{~nm}$ ) while N. Makarov was a graduate student at Montana State University. J. Campo acknowledges the Fund for Scientific Research of Flanders for a postdoctoral fellowship (Mobility Allowance FWO, Vlaanderen, Belgium). 